\title{
Les nitrates : origine et impact sur le milieu
}

\author{
Origins, evolution and impact of nitrates
}

\author{
par F. Dubois de la Sablonière
}

Agence de l'Eau Loire-Bretagne

Nitrates, an oxidized form of nitrogen, when found in water can have a natural origin. They can also be linked to human activity (industrial, domestic, agricultural).

Nitrates carried out to the natural environment are mostly diffuse and in weak quantity as compared to the amounts at stake or stored in the soil.

The transfer time towards water is quite varied according to the milieu: it may be of some days or some months. It might as well reach several decades at times.

Nitrates, although they are essential to the working of aquatic ecosystems, whenever in excess may entail eutrophication on the sea shore (proliferation of algae).

Furthermore, when they exceed $50 \mathrm{mg}$ per liter, surface water can no longer be wed for water supply because water used for domestic water supply must not be over $50 \mathrm{mg}$ per liter, whatever its origin.

All this leads to important environmental costs for the community, particularly as far as water supply treatment is concerned.

\section{I $\square$ ORIGINE DES NITRATES : LES QUANTITÉS MISES EN JEU}

L'ion nitrate est un dérivé essentiel de l'azote. Il représente le stade final de l'oxydation de l'azote et correspond à la forme assimilable principale de la nutrition azotée des végétaux.

Les nitrates ont plusieurs origines primaires :

- la fixation biologique,

- les précipitations atmosphériques,

- la minéralisation des résidus végétaux, animaux et de l'azote organique du sol.

Le développement des activités humaines a modifié le cycle naturel de l'azote en accroissant considérablement les quantités mises en jeu.

Les apports d'azote liés aux activités humaines peuvent être classés en trois grandes catégories d'après leur nature :

- l'activité industrielle avec des branches rejetant plus ou moins d'azote,

- l'activité d'élevage,

- l'activité agricole utilisatrice de fertilisants azotés.

\subsection{Données de base concernant l'azote}

Le tableau 1 présente les quantités d'azote mises en jeu (d'après rapport Henin 1980 réactualisé avec les données 1988 pour les effluents d'élevage et les données 1995 pour les engrais minéraux, à partir des travaux du SCEES).

Les productions d'effluents d'élevage et la consommation d'engrais minéraux sont globalement stables au niveau national depuis les années 1990.

\begin{tabular}{|l|c|}
\hline \multicolumn{1}{|c|}{ Origine de l'azote } & $\begin{array}{c}\text { Quantité d'azote libéré } \\
\text { ou apporté chaque année } \\
\text { (en millions de tonnes d'azote) }\end{array}$ \\
\hline Minéralisation des matières organiques du sol & 3,0 \\
\hline Engrais minéraux & 2,5 \\
\hline Apport atmosphérique & 0,5 \\
\hline Déjections humaines & 0,3 \\
\hline Déjections d'élevage & 1,3 \\
\hline Fixation symbiotique et non symbiotique & 1,3 \\
\hline Azote d'origine industrielle & 0,1 \\
\hline $\begin{array}{l}\text { Azote résultant du bilan importation-exportation } \\
\text { des produits agricoles (valeur négative) }\end{array}$ & 0,1 \\
\hline
\end{tabular}

Tableau 1. - Quantités d'azote mises en jeu en France. 


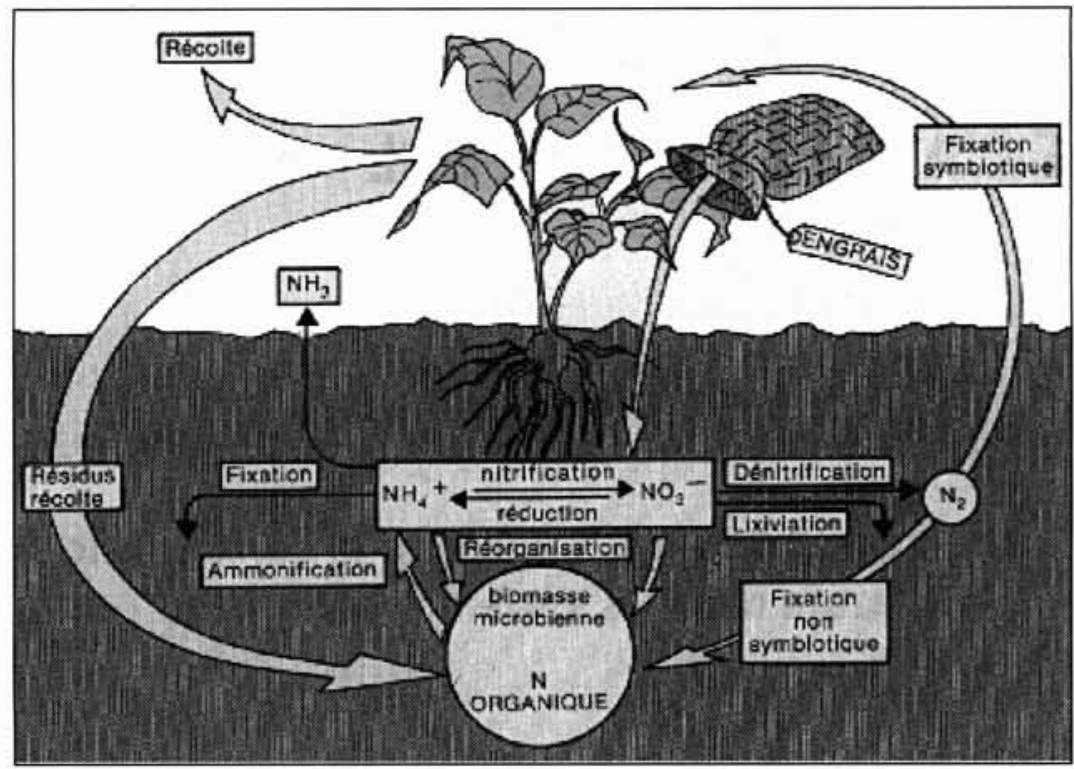

1. Le cycle de l'azote. D'après (Perspectives Agricoles juin 1997) Guiraud CEN Cadarache.

Par conséquent, chaque année, du fait des phénomènes spontanés d'une part, ou des activités humaines d'autre part, près de 9 millions de tonnes d'azote sont mises en jeu sous forme minérale dans le sol de notre pays. L'azote des engrais ne représente que $30 \%$ environ de cet ensemble.

Ces évaluations montrent que la maîtrise de la teneur des eaux en azote et plus particulièrement en nitrates doit porter sur une surveillance stricte de l'ensemble des processus concernant le cycle biologique de cet élément.

On estime que l'agriculture est responsable en France des deux tiers des apports aux eaux superficielles et que son rôle est déterminant dans la pollution des nappes.

Toutefois, il est montré que localement des apports domestiques peuvent générer des pics de pollution.

\section{II — L'ÉTAT DES MILIEUX}

\subsection{Les eaux de surface}

Les réseaux de mesure de bassin mis en place depuis 1991 permettent d'évaluer la qualité des eaux pour le paramètre nitrates.
Les résultats ci-dessous montrent la dégradation continue jusqu'à nos jours qui affecte principalement la grande moitié nord-ouest de la France, et principalement le nord (bassins Seine-Normandie et Artois-Picardie) et la Bretagne.

L'importance des activités agricoles dans cette contamination est tout à fait mise en évidence par le graphe de la figure 3 qui intègre deux bassins à typologie agricole très différente (la Vilaine, polyculture, élevage intensif et l'Allier, agriculture très diversifiée avec néanmoins beaucoup d'élevage extensif, sur herbages permanents).

\subsection{Les eaux souterraines}

De nombreuses données permettent de dresser la situation. Ce sont essentiellement les analyses réalisées par la DDASS dans le cadre du contrôle sanitaire des eaux. Toutefois cellesci donnent une vision biaisée puisque les captages les plus pollués, et à ce titre abandonnés, ne font plus l'objet d'un suivi. Le rapport Martin sur la gestion durable des eaux souterraines, de janvier 1996, fait état des éléments suivants :

“ Dès 1987, 15 à $20 \%$ des eaux souterraines captées pour les AEP avaient, semble-t-il, une teneur en nitrates supé-

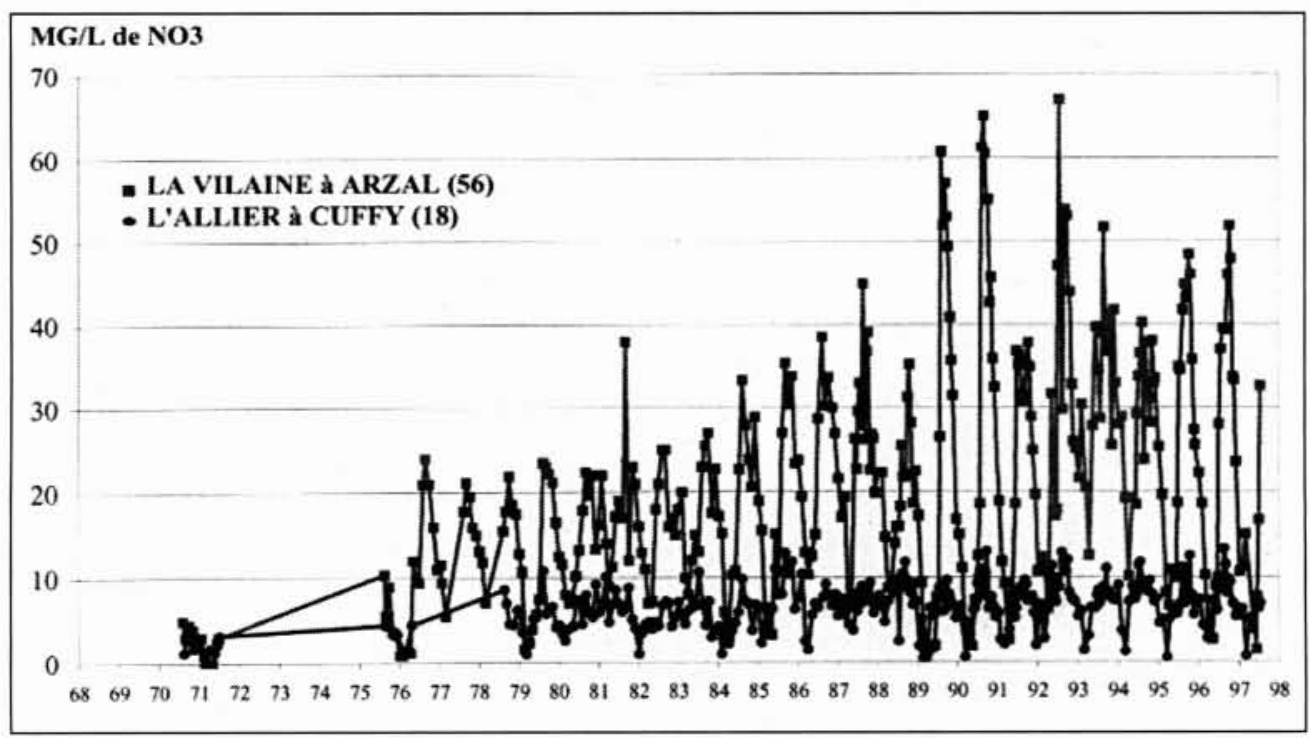

3. Evolution des concentrations en nitrates. Source : Agence de l'eau Loire-Bretagne 


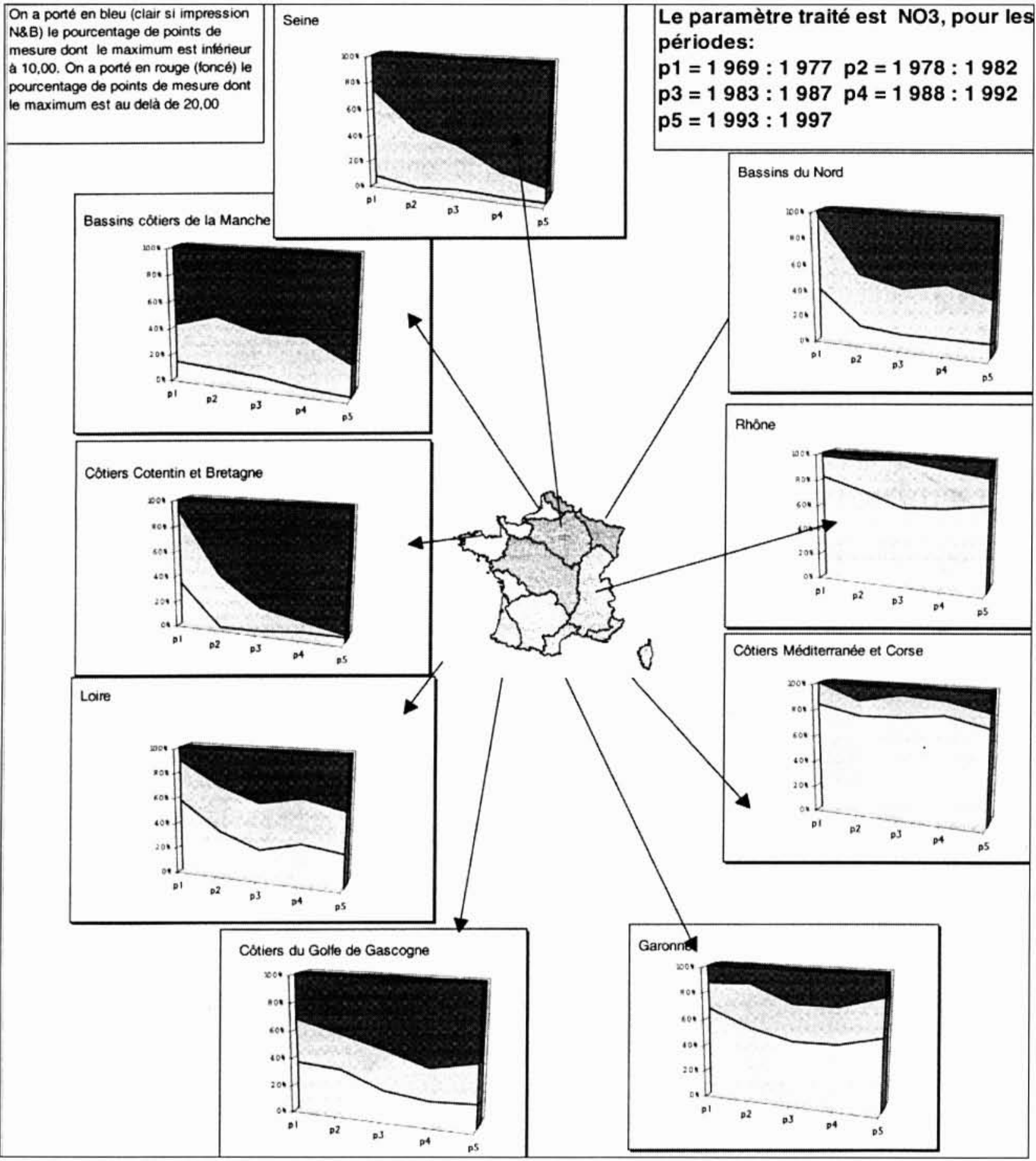

2. Dégradation des eaux de surface. Données RNDE, Agences de l'eau. Calcul IFEN.

4. Teneur en nitrate des eaux douces (points d'observation ayant servi à la délimitation des zones vulnérables).

Source : MATE - Direction de l'eau

N.B. Résultats établis à partir de l'analyse de 3300 points de captage répartis sur 89 départements. Le graphique se lit comme suit : 15 départements ont plus de $40 \%$ de leurs points de prélèvement qui dépassent la teneur de $40 \mathrm{mg} / \mathrm{l}$.

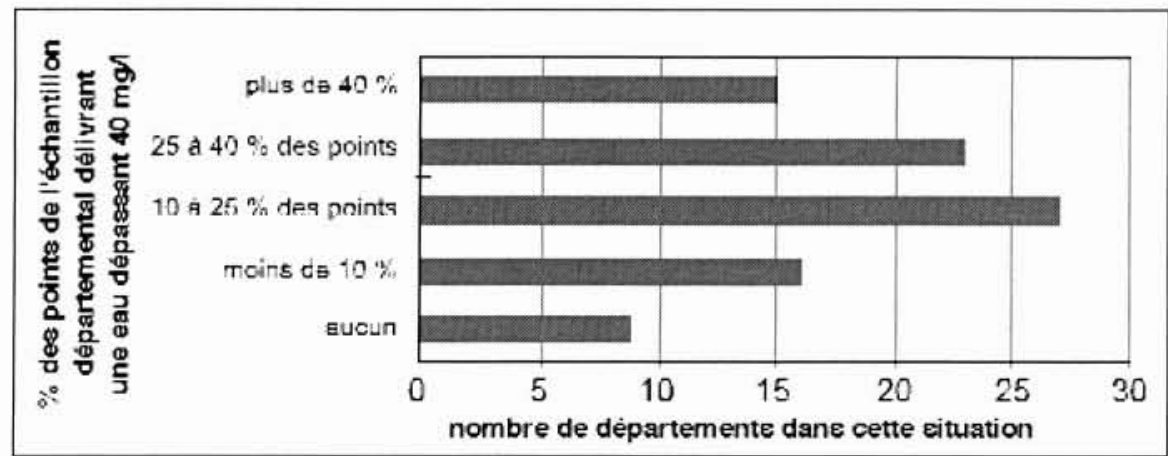




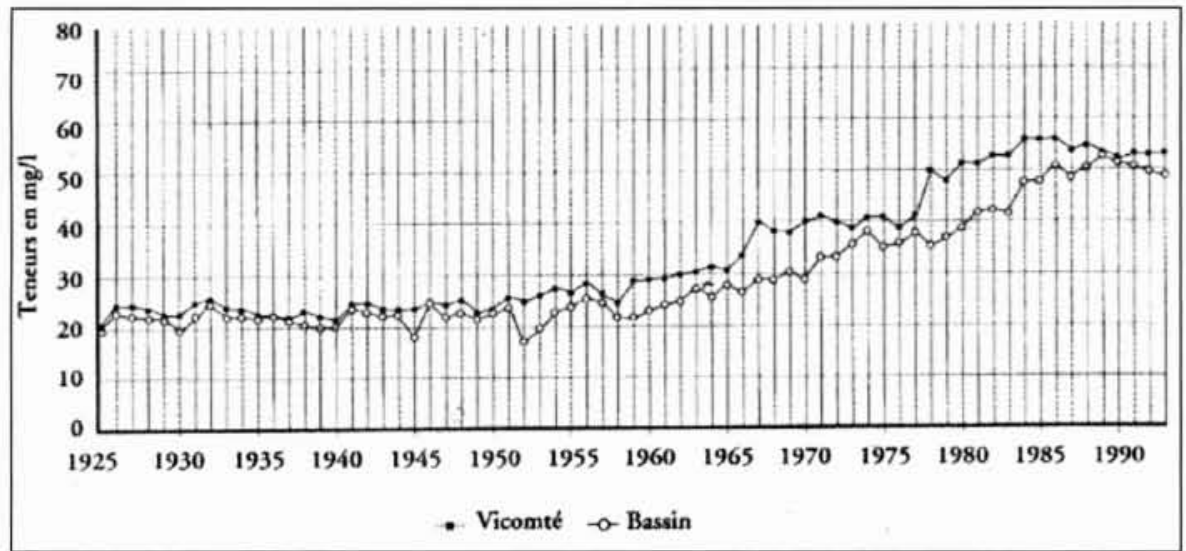

5. Evolution des nitrates - Source de la Voulzie. (Source SAGEP).
- un facteur lié à la quantité d'azote apportée par les eaux douces alimentant à plus ou moins brève échéance les eaux marines,

- un facteur lié à la sensibilité terrestre du bassin versant au risque de pollution (débits d'eau, nature du substrat pédologique et des sols, relief, capacité d'autoépuration du milieu, pluviométrie), - un facteur lié à la sensibilité du littoral (courants, effets de houle, température de l'eau, lumière, vent).

La lutte contre la prolifération des algues passe par la maîtrise des flux de nitrates au printemps. rieure à $50 \mathrm{mg} / \mathrm{l}$; un millier de forages auraient déjà été abandonnés pour cause de teneur excessive en nitrates et la concentration observée augmenterait de 1 à $2 \mathrm{mg} / \mathrm{l}$ par an (parfois même de $3 \mathrm{mg} / \mathrm{l}$ par an) dans les grandes nappes libres. Au rythme actuel, la moitié de nos prélèvements d'eau souterraine verraient leur concentration en nitrates dépasser $50 \mathrm{mg} / \mathrm{l}$ au début du XXI ${ }^{\mathrm{e}}$ siècle. Le chiffre de 200 captages abandonnés pour cause de pollution par les nitrates a été avancé à la suite d'une étude faite par le ministère de la Santé en 1988 (le rythme actuel des abandons serait de quelques dizaines) ".

La surveillance opérée du 1/09/92 au 31/08/93 dans le cadre de la directive nitrates et des programmes d'action en zones vulnérables, qui concerne plus du tiers du territoire national, laisse apparaître les résultats apparaissant sur les figures 4 et 5 .

\subsection{Les eaux marines littorales}

En 1997, plus de 45 sites littoraux bretons ont connu un phénomène d'accumulation significative d'algues vertes. On a pu observer, durant l'été 1997, 20000 tonnes d'ulves sur l'ensemble du littoral breton.

C'est sur le littoral des Côtes-d'Armor et du Finistère que le phénomène est le plus intense. La présence d'algues vertes sur les plages est une nuisance olfactive et visuelle qui touche les activités économiques du littoral, surtout le tourisme.

Les études menées par la communauté scientifique et les différents organismes concernés montrent que cette prolifération est due à la conjonction de 3 types de facteurs d'origine naturelle ou anthropique :

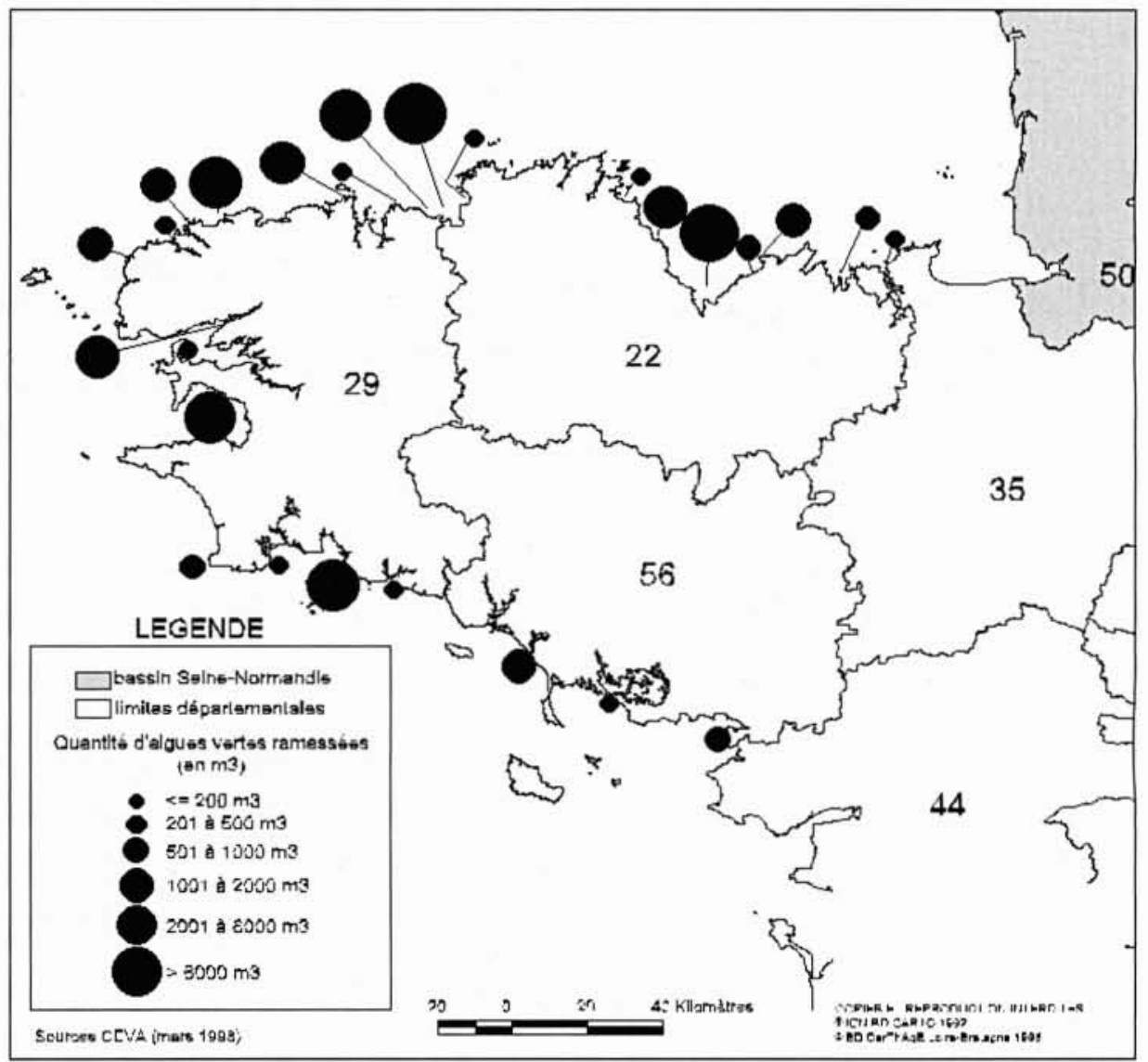

6. Quantité d'algues vertes ramassées sur le littoral breton. 
Tableau 2. - Bilan de potabilité des eaux distribuées dans le bassin Loire-Bretagne Dépassements chroniques (Source : DRASS Région Centre)

TENEUR MOYENNE ANNUELLE $>50 \mathrm{MG} / \mathrm{L}$

Etat 1989-1991 $\square$ Etat 1993-1994

\begin{tabular}{|l|c|c|c|c|c|c|}
\hline \multirow{2}{*}{ REgion } & \multicolumn{2}{|c|}{ Nombre U.D. } & \multicolumn{2}{c|}{$\begin{array}{c}\text { POPULATION } \\
\text { CONCERNEE }\end{array}$} & \multicolumn{2}{c|}{$\begin{array}{c}\text { POPULATION } \\
\text { TOTALE }\end{array}$} \\
\hline Auvergne & 2 & 1 & 4900 & 3400 & $0,4 \%$ & $0,3 \%$ \\
\hline Bourgogne & 4 & 1 & 3900 & 300 & $0,9 \%$ & $0,1 \%$ \\
\hline Bretagne & 36 & 25 & 57800 & 46600 & $2 \%$ & $1,6 \%$ \\
\hline Centre & 65 & 74 & 51200 & 82400 & $2,6 \%$ & $4,2 \%$ \\
\hline $\begin{array}{l}\text { Languedoc- } \\
\text { Roussillon }\end{array}$ & 0 & 0 & 0 & 0 & 0 & 0 \\
\hline Limousin & 0 & 2 & 0 & 600 & 0 & $0,1 \%$ \\
\hline Basse-Normandie & 0 & 3 & 0 & 500 & 0 & $0,3 \%$ \\
\hline Pays de la Loire & 11 & 21 & 8200 & 28100 & $0,2 \%$ & $0,9 \%$ \\
\hline Poitou-Charentes & 25 & 34 & 62300 & 124900 & $6,2 \%$ & $13,8 \%$ \\
\hline Rhône-Alpes & 5 & 5 & 700 & 700 & $0,1 \%$ & $0,1 \%$ \\
\hline Total & 148 & 166 & 189000 & 287500 & $1,6 \%$ & $2,4 \%$ \\
\hline
\end{tabular}

dantes desservies en 1994 par une eau contenant en moyenne plus de $50 \mathrm{mg} / \mathrm{l}$ de nitrates.

\section{III — LE COÛT DE LA POLLUTION PAR LES NITRATES}

Sont présentés ci-après des éléments de coûts relatifs à certaines actions curatives bien identifiées. D'autres actions dans le domaine de l'eau potable, comme la recherche et la mise en cuvre de ressource de substitution ou de dilution, sont également très onéreuses. Dans tous les cas, les actions préventives, rendues obligatoires par la réglementation (directive Nitrates...) sont indispensables à la protection du patrimoine naturel. Le coût des bonnes pratiques agricoles est estimé dans le rapport Martin à $800 \mathrm{MF} / \mathrm{an}$ si I'on inclut le coût de gestion de l'interculture.

\subsection{Coût de traitement des eaux destinées à la consommation humaine}

Deux procédés sont agréés en France :

- la dénitrification (1981) : procédé biologique qui repose sur la capacité des bactéries à dénitrifier et réduire les nitrates en azote gazeux ;

- la dénitratation par résines échangeuses d'ions (1985) : procédé physico-chimique (dans la grande majorité des usines) qui consiste à capter sur des résines les ions nitrates en échangeant une quantité équivalente d'ions dont la pré-

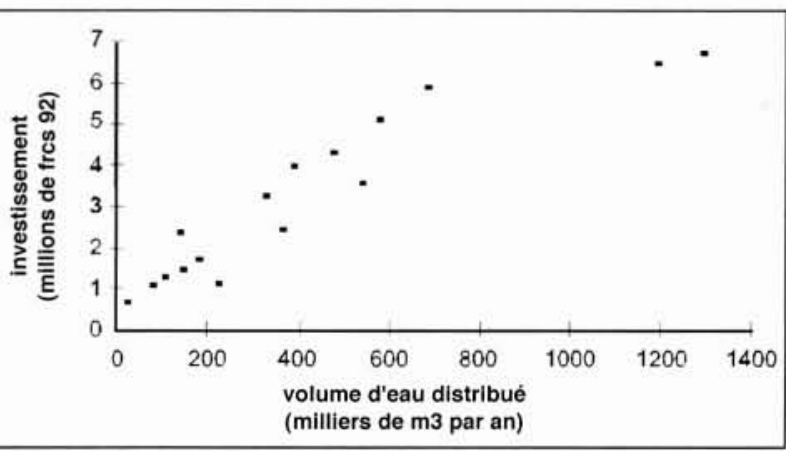

7. Coût d'investissement en fonction du volume annuel d'eau distribué. (Source INRA)

sence n'est pas gênante. Lors de la régénération de la résine on récupère une solution appelée éluat.

Une trentaine d'usines sont en fonctionnement, principalement dans les zones d'agriculture intensive (Bassin parisien, grand Ouest, vallée de la Garonne). Elles traitent essentiellement des eaux souterraines dont les teneurs en nitrates peuvent dépasser $75 \mathrm{mg} / \mathrm{l}$.

Ces usines, qui requièrent une technologie pointue, nécessitent un investissement élevé (cf. tableau 3).

Sur la base d'une consommation d'eau moyenne de 80 $\mathrm{m}^{3} /$ habitant et par an, ceci correspond à une dépense annuelle d'environ $140 \mathrm{~F} /$ habitant.

\begin{tabular}{|l|c|c|c|}
\hline & Amortissement & Fonctionnement & Total \\
\hline Procédé biologique & 0,64 & 0,86 & $\mathbf{1 , 5 0}$ \\
\hline Procédé physico-chimique & 0,92 & 0,80 & $\mathbf{1 , 7 2}$ \\
\hline Moyenne & 0,85 & 0,81 & $\mathbf{1 , 6 1}$ \\
\hline
\end{tabular}

Tableau 3. - Cô̂t de traitement des nitrates pour la fabrication d'eau potable. $\left(\mathrm{F} 92 / \mathrm{m}^{3}\right.$ d'eau distribuée) 


\subsection{Coût lié au développement algal sur le littoral}

La nuisance provoquée par le dépôt des algues sur le littoral a conduit les communes à mettre en œuvre un ramassage qui est, à l'heure actuelle, la seule action curative possible.

Dans le département des Côtes-d'Armor, $20000 \mathrm{~m}^{3}$ d'algues sont ramassées chaque année, pour un coût compris entre 1 et 1,5 MF. Les coûts de ramassage en fonction des sites, du matériel utilisé et des quantités ramassées, s'échelonnent entre 30 et $300 \mathrm{~F} / \mathrm{m}^{3}$.

Plus de la moitié des communes font appel à la mise en décharge pour globalement le tiers du volume ramassé ; cette solution devra être abandonnée en 2002 qui verra l'interdiction de mise en décharge.

L'autre destination est l'épandage sur des terres agricoles, mais la vulgarisation de la technique et son intégration dans les plans d'épandage (notamment en zone d'excédent structurel) posent localement problème.

\section{IV $\square$ CONCLUSION}

L'accroissement généralisé de la pollution des eaux par les nitrates traduit le déséquilibre du cycle de l'azote généré par l'intensification des activités agricoles et les modifications d'occupation des sols et de gestion de l'espace.
Cette pollution engendre un coût économique et un coût social se répercutant sur l'ensemble des acteurs économiques et à la fois sur les usages de l'eau marchands et non marchands.

Les études économiques, menées notamment par l'agence de l'eau Loire-Bretagne, montrent que les conséquences sur le prix de l'eau peuvent varier de quelques centimes à quelques francs $/ \mathrm{m}^{3}$. Elles montrent également que les activités économiques, comme la conchyliculture, I'industrie agro-alimentaire ou le tourisme, sont affectées par la dégradation de la ressource en eau en termes de chiffre d'affaires et d'emplois. Les solutions de protection préventive qui s'intègrent dans le cadre d'une gestion durable sont, à terme, moins onéreuses si l'on prend en compte la valeur patrimoniale.

\section{Bibliographie :}

[1] Rapport du groupe de travail activités agricoles et qualité des eaux . ministère de l'Agriculture - ministère de l'Environnement - 1980

[2] Agriculture et Environnement - Les indicateurs IFEN - 1997-1998

[3] Ministère de I'Industrie, de la Poste et des Télécommunications - rapport sur la gestion durable des eaux souterraines - 29/01/1996

[4] TSM - décembre 1995

[5] Bilan de potabilité des eaux distribuées dans le bassin Loire-Bretagne

[6] L'eau en Loire-Bretagne - juillet $96 n^{\circ} 57$ 